\title{
Photophysics of phycoerythrocyanins from the cyanobacterium Westiellopsis prolifica studied by time-resolved fluorescence and coherent anti-Stokes Raman scattering spectroscopy
}

\author{
S. Schneider ${ }^{a, *}$, W. Jäger ${ }^{a}$, C.-J. Prenzel ${ }^{a}$, G. Brehm ${ }^{a}$, P.S. Maruthi Sai ${ }^{a, 1}$, H. Scheer ${ }^{b}$, \\ F. Lottspeich \\ "Institut für Physikalische und Theoretische Chemie, Universität Erlangen, Egerlandstraße 3, D-91058 Erlangen, Germany \\ 'Botanisches Institut der Ludwig-Maximilians-Universität, Menzingerstr. 67, D-80638 Munich, Germany \\ 'Max-Planck-Institut für Biochemie, D-81375 Martinsried, Germany
}

Received 17 February 1994; accepted 2 May 1994

\begin{abstract}
Three building blocks of the antenna complexes of the cyanobacterium Westiellopsis prolifica were studied: $\mathrm{PEC}(\mathrm{X})$, which is similar to the $\alpha$-subunit of phycoerythrocyanin (PEC), trimers of PEC and monomers derived from these by deaggregation with KSCN. The fit of the fluorescence decay curve of $\mathrm{PEC}(\mathrm{X})$ requires at least four exponentials, although it supposedly contains only one chromophore. The coherent anti-Stokes Raman scattering (CARS) spectra indicate that the heterogeneity observed is due to geometrical isomers, which are in part generated by photoinduced processes. A similar heterogeneity in chromophore structure and properties is also found in the monomers, where four exponentials are needed to fit the fluorescence decay curve. As in trimers, there is a long-lived, low-amplitude component, which can be assigned to impurities and/or oxidation products. The energy transfer time between the two phyocyanobilin chromophores in the $\beta$-subunit is about 500 ps; the lifetime of the fluorescing $\beta$-chromophore is $1.5 \mathrm{~ns}$. The phycoviolobilin chromophore in the $\alpha$-subunit adopts different geometries characterized by fuorescence liftetimes of about 240 and 800 ps. No evidence was found for energy transfer between the $\alpha$ chromophore and the $\beta$-chromophores. This energy transfer occurs in trimers on a time scale of less than 20 ps; the energy transfer time between the two different types of $\beta$-chromophore is about 250 ps and the lifetime of the terminal emitter is about $1.5 \mathrm{~ns}$. The excited state kinetics are therefore similar to those of PEC trimers from Mastigocladus laminosus, as are the CARS spectra, indicating a similar chromophore-protein arrangement. In comparison with phycocyanin, the ordering of the excited states of chromophores $\beta 84$ and $\beta 155$ may be changed. Although PEC trimers of Westiellopsis prolifica show almost as good a photostability as trimers of Mastigocladus laminosus, monomers are so photolabile that no CARS spectra could be recorded.
\end{abstract}

Keywards: Phycoerythrocyanin; Fluorescence decay; Coherent anti-Stokes Raman scattering (CARS); Westiellopsis prolifica; Energy transfer

\section{Introduction}

Cyanobacteria are oxygen-evolving photosynthetic prokaryotes which form special light-harvesting complexes, the phycobilisomes [1]. These are composed of highly organized aggregates of trimers of up to three different biliproteins termed phycoerythrocyanin (PEC) or phycoerythrin (PE), phycocyanin (PC) and allophycocyanin (APC). Since the major absorption bands of these phycobiliproteins range between 450 and $660 \mathrm{~nm}$,

\footnotetext{
*Corresponding author.

'Present address: Department of Biochemistry, Weizmann Institute of Science, Rehovot, 76100 Israel.
}

they fill the gap in the absorption spectrum of chlorophyll $a$ which absorbs primarily in the blue (less than 450 $\mathrm{nm})$ and red $(650-700 \mathrm{~nm})$ regions of the visible spectrum. They increase the efficiency of light harvesting, and transfer the excitation energy to the photosynthetic reaction centres [2].

PEC is located at the periphery of the rod substructures of the phycobilisomes. It has been shown that the chromophores in PEC are tetrapyrroles as in PC, except that the $\alpha$-subunit of PEC carries a phycoviolobilin (PVB) chromophore instead of phycocyanobilin (PCB) [3] (see Fig. 1); X-ray studies have proven that the overall geometry of the chromophores in PEC is 


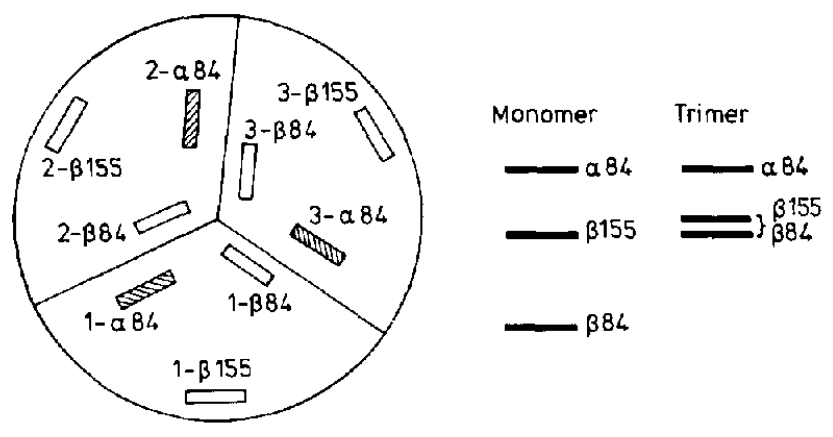

Fig. 1. Sketch of chromophore distribution (after Ref. [5]) and energy level scheme in monomeric and trimeric aggregates of PEC. 1- $\alpha 84$ is a phycoviolobilin chromophore bound to amino acid 84 of the $\alpha$ polypeptide chain of monomer $1 ; 1-\beta 84$ and $1-\beta 155$ are phycocyanobilin chromophores bound to amino acids 84 and 155 of the $\beta$ polypeptide chain of monomer 1 .
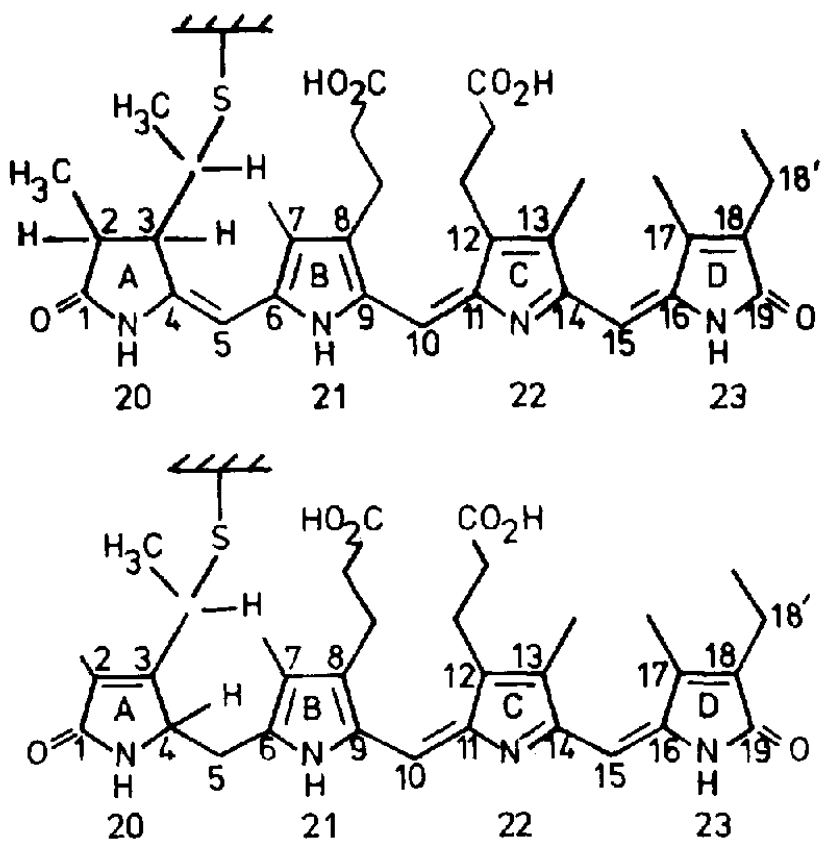

Scheme 1. Chemical structure of tetrapyrrole chromophores found in PEC: top, phycocyanobilin ( $\beta 84$ and $\beta 155$ ); bottom, phycoviolobilin (a84). Note that the configuration/conformation at the exocyclic double bonds is $5 Z$-s-anti, $10 Z$-s-syn, $15 Z$-s-anti.

similar to that of PC [4,5], except for the small differences which are induced by the substitution of the methine group by a methylene bridge between rings $\mathbf{A}$ and $\mathbf{B}$ (see Scheme 1). The reduction in the size of the conjugated $\pi$-electron system causes a hypsochromic shift of the long-wavelength absorption band of the $\alpha 84$ chromophore from about $618 \mathrm{~nm}$ in PC to about $570 \mathrm{~nm}$ in PEC.

Isolated PEC exhibits a reversible photochemistry similar to that in the native state. This property, which is reminiscent of the higher plant sensory receptor phytochrome [6], has been attributed to the $\alpha 84$ PVB chromophore [7-10]. Studies have also indicated that the extent of reversible photochemistry in PEC increases with decreasing size of the aggregate and vice versa $[7,9]$. This dual feature of light harvesting and reversible photochemistry makes PEC unique in the sense that it is probably the only structurally defined cyanobacterial biliprotein studied so far that exhibits reversible photochemistry in the native state. We believe, therefore, that it is worthwhile to extend the investigation of the photophysical and photochemical primary processes in this biliprotein, especially since, until recently, little information existed on the excited state kinetics of PEC, and only on PEC from a single organism, namely Mastigocladus laminosus [11-13]. Furthermore, most earlier studies have neglected the potential interference of photochemistry with energy transfer.

In this paper, we report the results of picosecond, time-resolved fluorescence spectroscopy and resonanceenhanced, coherent anti-Stokes Raman scattering (CARS) spectroscopy of trimers of PEC isolated from Westiellopsis prolifica ARM 365 and from a dissociation product called $\mathrm{PEC}(\mathrm{X})[10]$. This biliprotein is believed to be very similar to the $\alpha$-subunit and therefore provides the unique possibility to collect information on the chromophore in this subunit. The results are discussed in comparison with those obtained earlier for PEC from Mastigocladus laminosus [11-15].

\section{Materials and methods}

Westiellopsis prolifica was obtained as an agar slant from the National Facility for Blue-Green Algae, Indian Agricultural Research Institute, New Delhi, India, and was grown in batch cultures of 5-10 1 in BG-11 medium as described previously [9]. Intact phycobilisomes were isolated according to a new procedure [9]. Two PEC fractions were obtained from the isolated phycobilisomes after dissociating the latter by dialysis at $4{ }^{\circ} \mathrm{C}$ against potassium phosphate buffer $(5 \mathrm{mM}, \mathrm{pH} 7.0)$. The dissociated phycobilisomes were applied onto diethylaminoethyl (DEAE) cellulose (Whatman, DE52) columns, and the constituent biliproteins were eluted with a step gradient (in approximately $10 \mathrm{mM}$ steps up to $200 \mathrm{mM}$ ) of potassium phosphate buffer ( $\mathrm{pH} 7.0$ ). The first fraction was PEC(X) and the second trimeric PEC. Monomers of PEC were obtained by addition of KSCN (1 M) to the PEC trimers [15].

Steady state absorption spectra were recorded on a Hewlett-Packard 8451A diode array spectrophotometer. The steady state fluorescence excitation and emission spectra were recorded on a Spex Fluorolog 221 spectrophotometer with $90^{\circ}$ geometry. All spectra were corrected for instrument response.

Fluorescence decay times were determined using a standard single-photon-counting apparatus [16]. Excitation was performed at $575 \mathrm{~nm}$ with the output of a synchronously pumped, mode-locked, cavity-dumped 
dye laser. The fluorescence was spectrally dispersed by a prism monochromator (PMQ 2). Using a selected R928 Hamamatsu photomultiplier and paying special attention to correct focusing of the fluorescence on the photocathode, an instrument response time (full width at half-maximum (FWHM)) of approximately $300 \mathrm{ps}$ was achieved. The usual multi-exponential, least-squares analysis was applied to the individual decay curves recorded for different detection wavelengths (time increment per channel, 13.9 ps; number of channels employed, 1700; number of counts at maximum, 5000).

The experimental apparatus for recording the CARS spectra [17] used the output of two dye lasers which were focused onto the cooled sample cuvette at a fixed angle $\left(2-5^{\circ}\right)$. The CARS beam was directed into a double monochromator for separation of stray light and recorded using a gated integrator. The pulse intensities were about $1 \mathrm{~mJ}$ (pump pulse) and $0.5 \mathrm{~mJ}$ (Stokes pulse); the pulse duration was approximately $15 \mathrm{~ns}$. In order to reduce photoinduced damage, the repetition rate of the laser pulse was lowered to about $3 \mathrm{~Hz}$; each data point in the spectrum is the average of 25 laser shots. The full lines shown in the spectra are the best fits based on the expression for the third- order susceptibility $\chi^{(3)}$

$\left|\chi^{3}\right|^{2} \propto\left|B+\sum_{r} \frac{R_{\mathrm{r}}+i I_{\mathrm{r}}}{\delta_{\mathrm{r}}-i \Gamma_{\mathrm{r}}}\right|^{2}$

where $B$ is the non-resonant background, $R_{\mathrm{r}}$ and $I_{\mathrm{r}}$ are the real and imaginary parts of the resonance-enhanced contribution due to the molecular vibration $\nu_{\mathrm{r}}, \Gamma_{\mathrm{r}}$ is the Raman linewidth of this vibration and $\delta_{\mathrm{r}}=\nu_{\mathrm{r}}-\left(\nu_{\mathrm{p}}-\nu_{\mathrm{s}}\right)$ where $\nu_{\mathrm{r}}, \nu_{\mathrm{p}}$ and $\nu_{\mathrm{s}}$ are the wavenumbers of the molecular vibration, pump and Stokes laser respectively. Depending on the ratio between $I_{\mathrm{r}}$ and $R_{\mathrm{r}}$, the line shape can vary between lorentzian and left or right dispersive. For this reason, band maxima do not necessarily coincide with the positions of the molecular frequencies, but can be shifted. The numbers given in the figures represent the true vibrational frequencies as derived by the fitting routine [17]. Lists of all fitting parameters (frequencies $\nu_{\mathrm{r}}$, linewidths $\Gamma_{\mathrm{r}}$, relative intensities $\left.A_{\mathrm{r}}=\left(R_{\mathrm{r}}^{2}+I_{\mathrm{r}}^{2}\right)^{1 / 2}\right)$ are available as supplementary material (see also below).

\section{Results}

\subsection{Absorption and stationary fluorescence}

The absorption and fluorescence emission spectra of PEC (X) and PEC monomers and trimers of Westiellopsis prolifica are shown in Figs. 2 and 3. They have been discussed in detail previously [9], and therefore we only need to summarize those features which are essential

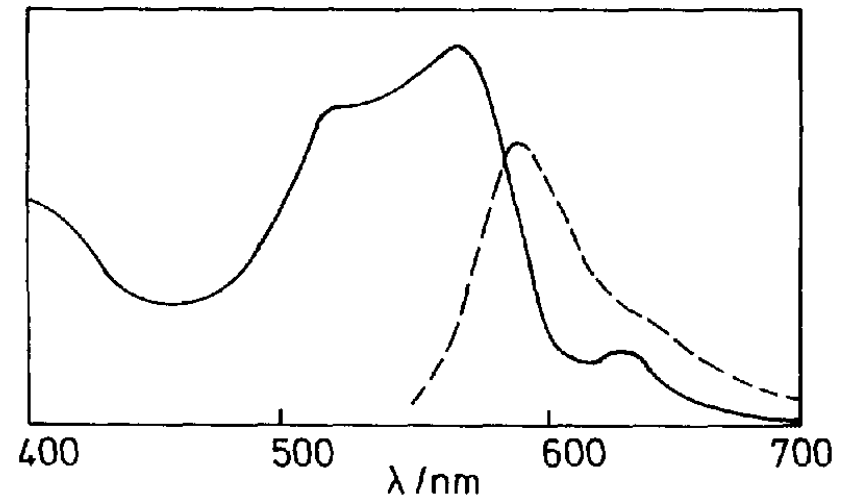

Fig. 2. Absorption (straight line) and fluorescence (dashed line) spectra $\left(\lambda_{e x}=540 \mathrm{~nm}\right)$ of $\operatorname{PEC}(X)$ in potassium phosphate buffer (50 $\mathrm{mM}, \mathrm{pH}$ 7.0).

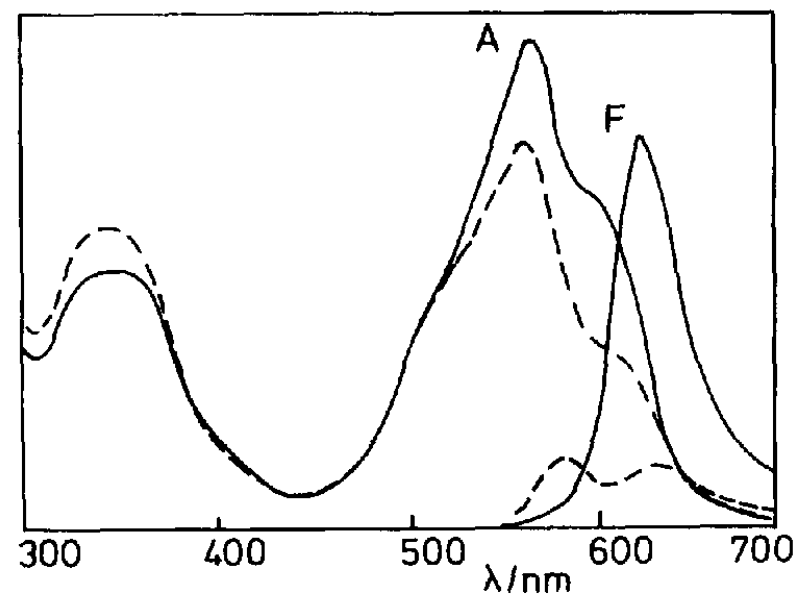

Fig. 3. Absorption (A) and fluorescence (F) spectra $\left(\lambda_{\mathrm{ex}}=540 \mathrm{~nm}\right.$ ) of PEC trimers (dashed line) and monomers (straight line) in potassium phosphate buffer $(100 \mathrm{mM}, \mathrm{pH} 7.0)$. Monomerization by addition of $1 \mathrm{M} \mathrm{KSCN}$.

for the discussion of our time-resolved fluorescence data. The absorption spectrum of $\operatorname{PEC}(X)$ is similar to that of the $\alpha$-subunit of PEC from Mastigocladus laminosus [10]. It exhibits a maximum around $565 \mathrm{~nm}$ and a pronounced shoulder around $510 \mathrm{~nm}$ (Fig. 2). In accordance with the absence of PCB chromophores, the fluorescence emission spectrum of $\mathrm{PEC}(\mathrm{X})$ has a maximum around $590 \mathrm{~nm}$ and is independent of the excitation wavelength in the range $540-565 \mathrm{~nm}$. The $510 \mathrm{~nm}$ shoulder originates probably (as in the case of PEC from Mastigocladus laminosus [7,8]) from an isomer which is derived from the $565 \mathrm{~nm}$ absorbing form by isomerization around the $\mathrm{C} 15-\mathrm{C} 16$ double bond. Since PEC(X) shows pronounced photochromic behaviour [8], the formation of this $510 \mathrm{~nm}$ isomer is obviously one of the photochemical decay channels of the $565 \mathrm{~nm}$ absorbing form. The fluorescence yield of the $510 \mathrm{~nm}$ form is extremely low [9]. For this and experimental reasons, we restricted our time-resolved fluorescence measurements to an excitation wavelength 
of $575 \mathrm{~nm}$, which is in all cases close to the absorption maximum of the $\alpha$-chromophore in its predominant form. The absorption peak around $630 \mathrm{~nm}$ is due to impurities. Consequently, there are doubts as to whether the shoulder in the fluorescence spectrum around 650 $\mathrm{nm}$ is also due to impurity fluorescence.

Trimeric PEC is stable in $100 \mathrm{mM}$ potassium phosphate buffer ( $\mathrm{pH} 7.0$ ). Its absorption spectrum is similar to that of trimeric PEC from other species $[7,8,15]$ with $\lambda_{\max } \approx 575 \mathrm{~nm}$ and a pronounced shoulder around 605 nm (Fig. 3, full line). Excitation in the range of the PVB chromophore absorption (540-575 $\mathrm{nm}$ ) results (independent of excitation wavelength) in an emission with $\lambda_{\max } \approx 630 \mathrm{~nm}$. This emission originates undoubtedly from the PCB chromophores and indicates that energy transfer from the PVB chromophore in the $\alpha$ subunit to the PCB chromophores in the $\beta$-subunit is very efficient.

Monomerization by addition of $\mathrm{KSCN}(1 \mathrm{M})$ induces minor changes in the absorption spectrum (Fig. 3, broken line). The absorbance is increased in the blue, but decreased in the red with a concomitant apparent shift of the shoulder by a few nanometers. With $540 \mathrm{~nm}$ excitation, the stationary fluorescence is strongly decreased and the spectrum shows two maxima. The first maximum $(590 \mathrm{~nm})$ is assigned to emission from the $\alpha$-chromophore and the second $(640 \mathrm{~nm})$ to emission from the PCB chromophores of the $\beta$-subunit. It was concluded that no or only inefficient energy transfer occurs from the PVB chromophore to the PCB chromophores in the monomeric unit. Similar observations have been made in PEC from Mastigocladus laminosus monomerized by addition of KSCN [7]. There, however, the short- wavelength emission is absent if monomerization is performed using urea [18].

\subsection{Time-resolved fluorescence measurements}

As mentioned previously, excitation of fluorescence was performed at $575 \mathrm{~nm}$ in all three samples. For PEC(X), the fitting parameters (Table 1) show an interesting dependence on observation wavelength. All

Table 1

Dependence of fitting parameters of fluorescence decay curves on detection wavelength for $\mathrm{PEC}(\mathrm{X})$ of Westiellopsis prolifica $\left(\lambda_{\mathrm{cx}}=575\right.$ nm)

\begin{tabular}{llllllll}
\hline$\lambda$ & $\begin{array}{l}\tau_{1} \\
(\mathrm{~nm})\end{array}$ & $\begin{array}{l}\tau_{2} \\
(\mathrm{~ns})\end{array}$ & $\begin{array}{l}\tau_{3} \\
(\mathrm{~ns})\end{array}$ & $\begin{array}{l}\boldsymbol{A}_{1} \\
(\%)\end{array}$ & $\begin{array}{l}\boldsymbol{A}_{2} \\
(\%)\end{array}$ & $\begin{array}{l}A_{3} \\
(\%)\end{array}$ & $\chi^{2}$ \\
\hline 590 & 0.15 & 0.7 & 2.9 & 75 & 21 & 4 & 1.07 \\
600 & 0.17 & 0.9 & 2.9 & 63 & 25 & 12 & 0.98 \\
610 & 0.20 & 1.3 & 3.9 & 61 & 28 & 11 & 1.08 \\
620 & 0.21 & 1.5 & 5.4 & 65 & 29 & 6 & 1.20 \\
630 & 0.20 & 1.5 & 6.2 & 61 & 30 & 9 & 1.29 \\
640 & 0.18 & 1.5 & 6.4 & 58 & 30 & 12 & 1.22 \\
650 & 0.19 & 1.5 & 6.3 & 56 & 33 & 11 & 1.26 \\
\hline
\end{tabular}

three decay times increase when the detection wavelength is varied from 590 to $620 \mathrm{~nm}$. Above $620 \mathrm{~nm}$, the decay times become independent of observation wavelength. The amplitude of the fast decay component decreases from about $75 \%$ to $56 \%$ with increasing detection wavelength, while the amplitude of the second component increases from $21 \%$ to $33 \%$. The amplitude of the third component appears to be fairly independent of detection wavelength and contributes generally around $10 \%$ in relative amplitude. These variations in decay times and amplitude indicate, in our opinion, that the three-exponential fit is descriptive rather than a true image of the excited state kinetics. It can reflect the fact that there is actually a wide distribution of chromophore geometry with the maximum emission occuring at different wavelengths and with different decay times. For an easier comparison with the results for PEC monomers and trimers, the variation of the relative amplitudes of these three components is presented in graphic form in Fig. 4(A).

The fits of the fluorescence decay curves of PEC trimers require four exponentials, one of which has a negative amplitude. Since the corresponding rise time is very fast, it is below the time resolution of our apparatus. This implies that the magnitude of its calculated amplitude is of no real physical meaning (it is subject to errors which are too large) and it is therefore omitted in Table 2. In contrast with PEC(X), we can claim that the decay times derived for the three decay components are fairly independent of observation wavelength, but their relative amplitudes change (Fig. 4(B)). The fastest decay time is calculated to be within 200 and $300 \mathrm{ps}$, the decay time of the second component ranges between 1 and $1.3 \mathrm{~ns}$ and the longest lived species exhibits a decay time around 2.5 ns. Due to its low amplitude, the decay time of the latter could be subject to a larger error at the detection wavelength of $610 \mathrm{~nm}$ or below. There is again a unique trend in the amplitude of the first and second decay components, similar to that observed for PEC(X). When the observation wavelength is increased from 590 to $630 \mathrm{~nm}$, the amplitude of the fast component decreases by nearly $50 \%$, whereas the amplitude of the second component increases by roughly a factor of two. The third, longer lived component shows an amplitude of about $10 \%$ independent of the observation wavelength (see Fig. 4(B)).

The fits of the fluorescence decay curves of the monomers also require at least four exponentials. However, in contrast with the trimers, no rising component is observed. Table 3 presents the decay times and relative amplitudes of all four decay components. Since the amplitude of the fourth component with a decay time above 3 ns is fairly low, we can speculate whether or not this component is due to impurities and/or oxidation products. If we restrict the discussion to the 

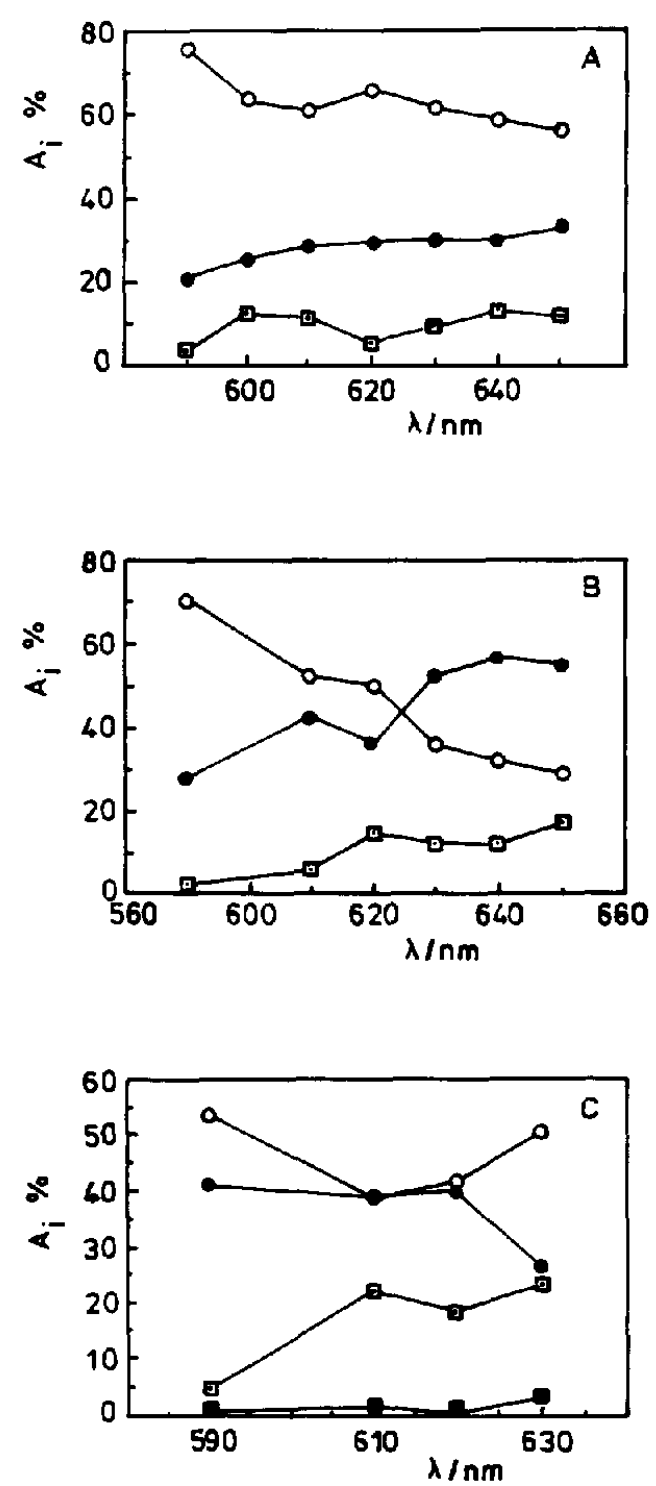

Fig. 4. Variation in relative amplitude of the various components in the multi-exponential fit of the fluorescence decay curves with detection wavelength. (A) PEC(X): $\tau_{1}\left(150-200 \mathrm{ps)}(\mathrm{O}) ; \tau_{2}(700-1500\right.$ ps) (๑); $\tau_{3}(2.8-6.2 \mathrm{~ns})$ (प). (B) PEC (trimer): $\tau_{\mathrm{t}}(200-300 \mathrm{ps})(\mathrm{O})$; $\tau_{2}(1.0-1.3 \mathrm{~ns})(\Theta) ; \tau_{3}(2-4 \mathrm{~ns})(\square)$. (C) PEC (monomer): $\tau_{1}(27-240$ ps) $(0) ; \tau_{2}(400-800 \mathrm{ps})(\ominus) ; \tau_{3}(1.3-2.0 \mathrm{~ns})(\square) ; \tau_{1}(3-6 \mathrm{~ns})(\square)$.

remaining three components, we observe a general pattern which is slightly different from that described above for the trimers (Fig. 4(C)). Although for detection wavelengths of 610 and $620 \mathrm{~nm}$ the fitting parameters are fairly close to each other, we obtain significant deviations when fitting the decay curve recorded at 590 $\mathrm{nm}$ (increase in decay times by a factor of two for the first and second components and low amplitude of the third component). With $630 \mathrm{~nm}$ detection, the decay time of the first component is at the limit of our time resolution ( $27 \mathrm{ps})$. The large differences in the decay times derived for observation wavelengths of 590 and $610 \mathrm{~nm}$ can be rationalized on the basis of the static fluorescence emission spectra. At $590 \mathrm{~nm}$, the emission
Table 2

Dependence of fitting parameters of fluorescence decay curves on detection wavelength for trimers of Westiellopsis prolifica $\left(\lambda_{\mathrm{ex}}=575\right.$ $\mathrm{nm})$. The fits require a fourth component $\left(A_{1}, \tau_{1}\right)$ with negative amplitude which is omitted in the table (for more details, see text)

\begin{tabular}{llllllll}
\hline$\lambda$ & $\tau_{2}$ & $\tau_{3}$ & $\tau_{4}$ & $A_{2}$ & $A_{3}$ & $A_{4}$ & $x^{2}$ \\
$(\mathrm{~nm})$ & $(\mathrm{ns})$ & $(\mathrm{ns})$ & $(\mathrm{ns})$ & $(\%)$ & $(\%)$ & $(\%)$ & \\
\hline 590 & 0.21 & 1.0 & 2.7 & 70 & 28 & 2 & 1.13 \\
610 & 0.25 & 1.2 & 2.8 & 52 & 43 & 5 & 1.16 \\
620 & 0.21 & 1.1 & 2.1 & 50 & 36 & 14 & 1.17 \\
630 & 0.28 & 1.3 & 2.4 & 36 & 52 & 12 & 1.10 \\
640 & 0.29 & 1.3 & 2.4 & 32 & 57 & 11 & 1.19 \\
650 & 0.27 & 1.3 & 2.3 & 29 & 55 & 16 & 1.17 \\
\hline
\end{tabular}

of the PVB chromophore should dominate the decay curve, whereas at 610 and $620 \mathrm{~nm}$, the PCB chromophores should be prominent.

\subsection{Coherent anti-Stokes Raman scattering spectra}

In the past, we have shown [19] that CARS is a valuable tool for comparing biliproteins originating from different organisms. It has been shown that, for PC, there are pronounced differences in the CARS spectra, especially in the fingerprint region. These differences are more pronounced for trimers than for monomers, indicating that there are changes in the chromophore-protein arrangement on aggregation which are different in the PCs of Mastigocladus laminosus and Westiellopsis prolifica. In order to provide a basis for a comparative discussion of the fluorescence kinetics of PEC originating from Mastigocladus laminosus and Westiellopsis prolifica, we recorded CARS spectra of PEC trimers of Westiellopsis prolifica with pump wavelengths of 585 and $630 \mathrm{~nm}$. They are shown in Fig. 5 for the range $1200-1700 \mathrm{~cm}^{-1}$. For technical reasons (constant crossing angle of pump and Stokes beam [17]), sections of only $100-150 \mathrm{~cm}^{-1}$ could be recorded without changing the experimental conditions. For $\mathrm{PEC}(\mathrm{X})$ we succeeded in recording CARS spectra only in the fingerprint and double bond stretching region because of rapid bleaching. In the spectral region between 1300 and $1550 \mathrm{~cm}^{-1}$, where the bands are very weak in the spectra of the trimers, the signal intensity was too low. For comparison, we have also included in Fig. 5 the spectra of PEC trimers from Mastigocladus laminosus [14] recorded with the same two pump wavelengths. In contrast with monomers from Mastigocladus laminosus, the monomers of Westiellopsis prolifica were so photolabile that no spectra could be recorded with either pump wavelength. This is in accordance with the general observation that PEC from Westiellopsis prolifica shows a larger photochromic effect, and is also more readily bleached.

The trimer CARS spectra recorded with long-wavelength pump radiation should be very close to each 
Table 3

Dependence of fitting parameters of fluorescence decay curves on detection wavelength for monomers of Westiellopsis prolifica $\left(\lambda_{e x}=575 \mathrm{~nm}\right.$ )

\begin{tabular}{lllllllll}
\hline $\begin{array}{l}\lambda \\
(\mathrm{nm})\end{array}$ & $\begin{array}{l}\tau_{1} \\
(\mathrm{~ns})\end{array}$ & $\begin{array}{l}\tau_{2} \\
(\mathrm{~ns})\end{array}$ & $\begin{array}{l}\tau_{3} \\
(\mathrm{~ns})\end{array}$ & $\begin{array}{l}\tau_{4} \\
(\mathrm{~ns})\end{array}$ & $\begin{array}{l}A_{1} \\
(\%)\end{array}$ & $\begin{array}{l}A_{2} \\
(\%)\end{array}$ & $\begin{array}{l}A_{3} \\
(\%)\end{array}$ & $\begin{array}{l}A_{4} \\
(\%)\end{array}$ \\
\hline 590 & 0.24 & 0.8 & 1.9 & 6.2 & 53 & 40.8 & 5 & 2 \\
610 & 0.14 & 0.48 & 1.3 & 5.0 & 39 & 39 & 21 & 1.14 \\
620 & 0.14 & 0.60 & 1.6 & 5.1 & 41 & 40 & 18 & 1.14 \\
630 & 0.03 & 0.40 & 1.3 & 3.1 & 50 & 25 & 22 & 3 \\
\hline
\end{tabular}
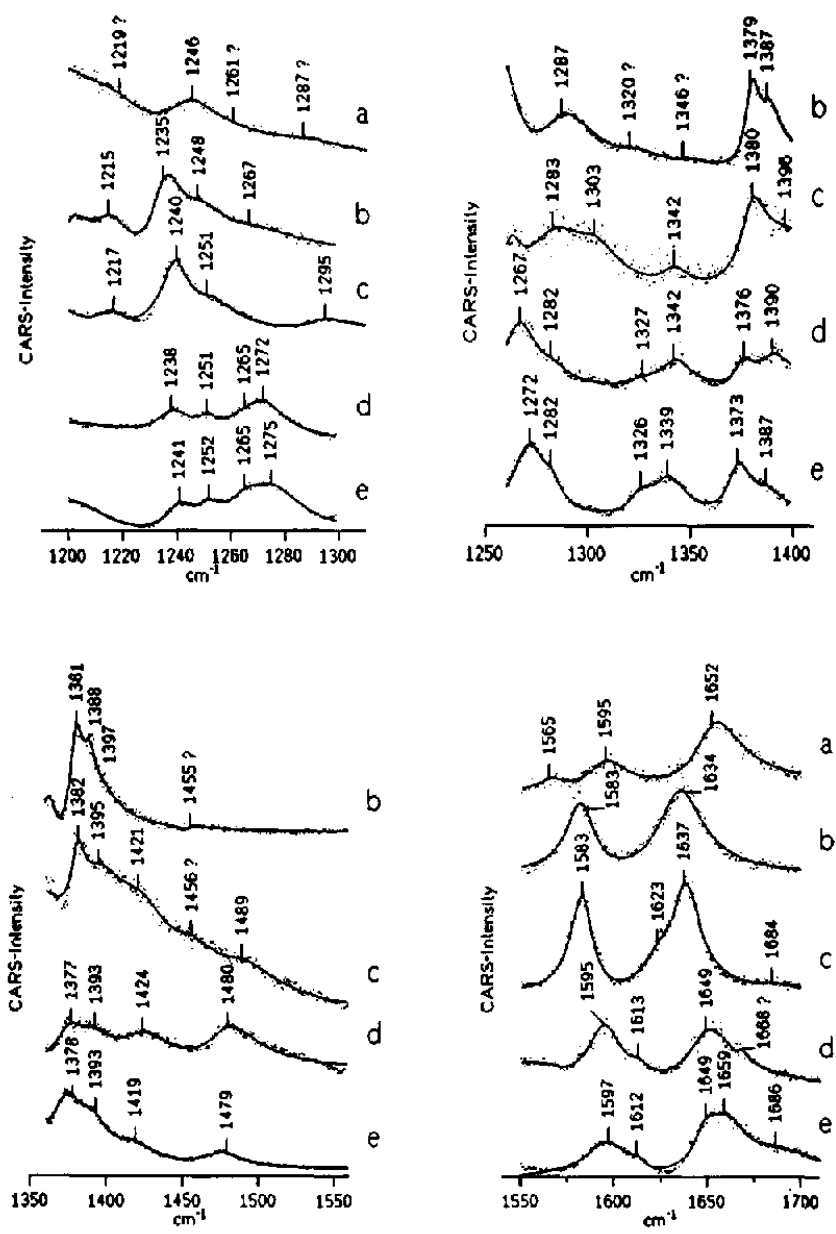

Fig. 5. Comparison of CARS spectra of $\operatorname{PEC}(X)$ (traces a) and trimers of PEC from Westiellopsis prolifica (traces $\mathrm{c}$, d) with trimers of PEC from Mastigocladus laminosus (traces b, e). Pump wavelength was $585 \mathrm{~nm}$ (traces a, b, c) and $630 \mathrm{~nm}$ (traces d, e).

other if the PCB chromophores exhibit the same chromophore-protein arrangement in both Mastigocladus laminosus and Westiellopsis prolifica. A comparison of the $630 \mathrm{~nm}$ spectra displayed in Fig. 5 (traces d and e) confirms that the spectra are indeed very similar in most features. In general, there is very good agreement between the vibrational frequencies derived by the fitting routine. There are, however, distinct differences in intensity distribution. The bands observed at 1201, 1265, 1327 and $1376 \mathrm{~cm}^{-1}$ are decreased in relative intensity in Westiellopsis prolifica compared with those in Mastigocladus laminosus, whereas the bands around 1424, 1480 and $1595 \mathrm{~cm}^{-1}$ are relatively stronger than in Mastigocladus laminosus. Furthermore, there is a shoulder around $1686 \mathrm{~cm}^{-1}$ in Mastigocladus laminosus which is absent in Westiellopsis prolifica.

More dramatic than the differences in the $630 \mathrm{~nm}$ spectra of Mastigocladus laminosus and Westiellopsis prolifica are, however, the changes which are observed in both species with a change in pump wavelength to $585 \mathrm{~nm}$ (Fig. 5, traces $b$ and c). A new band around $1215 \mathrm{~cm}^{-1}$ arises in the spectra of both species, but the most prominent band in the fingerprint region is found around $1240 \mathrm{~cm}^{-1}$. The $1265 \mathrm{~cm}^{-1}$ band is fairly weak and that at $1275 \mathrm{~cm}^{-1}$ is completely missing in the $585 \mathrm{~nm}$ spectra. In the double bond stretching region the shoulder around $1612 \mathrm{~cm}^{-1}$ has disappeared, although the most intense feature around $1595 \mathrm{~cm}^{-1}$ is shifted by about $12 \mathrm{~cm}^{-1}$ to $1583 \mathrm{~cm}^{-1}$. The broad (double) band around $1650 \mathrm{~cm}^{-1}$ appears around 1637 $\mathrm{cm}^{-1}$ with a shoulder around $1623 \mathrm{~cm}^{-1}$. Major differences between the 630 and $585 \mathrm{~nm}$ spectra are also observed in the spectral region between 1270 and 1500 $\mathrm{cm}^{-1}$. Despite the larger scatter in the data points and the weakness of many bands, several statements can be made beyond doubt. One concerns the appearance of a weak band around or above $1300 \mathrm{~cm}^{-1}$ and the reduction in intensity of the bands around 1340 and $1480 \mathrm{~cm}^{-1}$ in the $585 \mathrm{~nm}$ spectra. Furthermore, there is a difference in the appearance of the spectra in the region $1250-1300 \mathrm{~cm}^{-1}$, which is covered in the two sections presented at the top of Fig. 5 .

It should be mentioned that, in PEC trimers of Mastigocladus laminosus, we also observe changes in relative intensity on illumination of the sample with green light. The obvious explanation [14] is that, due to the intense irradiation which is necessary to record the CARS spectra, photoisomerization of the $\alpha$-chromophore is induced, with the effect that after a short time of measurement the samples contain an increased amount of the $510 \mathrm{~nm}$ absorbing species. If we then irradiate continuously with $514.5 \mathrm{~nm}$ laser light, a major fraction of the photochemistry is reversed and the signal contribution from species absorbing around $575 \mathrm{~nm}$ is increased. Among those bands which show a significant 
variation in intensity on green light irradiation is the band around $1295 \mathrm{~cm}^{-1}$, which is present in the first section (Fig. 5, top left) of the $585 \mathrm{~nm}$ spectrum of Westiellopsis prolifica, but missing in that of Mastigocladus laminosus.

The CARS spectra of $\operatorname{PEC}(\mathrm{X})$ show a low signalto-noise ratio for several reasons. Firstly, the optical density (OD) of the samples at the pump wavelength is low due to limitations in the availability of the material $\left(O D<4 \mathrm{~cm}^{-1}\right)$. Furthermore, the intensities of the 585 nm pump and Stokes beams had to be reduced to minimize photoinduced denaturation (aggregation and sedimentation). Nevertheless, we can identify a band at $1246 \mathrm{~cm}^{-1}$ and weak features around 1219 and 1287 $\mathrm{cm}^{-1}$. In the double bond stretching region, the PEC(X) spectrum has nothing in common with the $585 \mathrm{~nm}$ trimer spectrum. The most intense band is found around $1652 \mathrm{~cm}^{-1}$. This frequency is very close to that of one band maximum found for PEC trimers with a pump wavelength of $630 \mathrm{~nm}$. The position of the second most intense band also agrees well with that of the second most intense band in the $630 \mathrm{~nm}$ spectrum. The third band at $1565 \mathrm{~cm}^{-1}$ is certainly absent in the $585 \mathrm{~nm}$ spectrum, but is indicated as a weak feature in the $630 \mathrm{~nm}$ spectrum. From these differences we must conclude that the protein-chromophore arrangement in $\operatorname{PEC}(\mathrm{X})$ is different from that of the $\alpha$-subunit in the PEC trimers, at least for strong irradiation with light wavelengths around $585 \mathrm{~nm}$. Under these conditions, several features are closer to those observed in the spectra of the PCB chromophores.

\section{Discussion}

The large similarity between the CARS spectra of the PEC trimers from Mastigocladus laminosus and Westiellopsis prolifica, recorded with $630 \mathrm{~nm}$ pump radiation, suggests that the overall chromophore-protein arrangement in the $\beta$-subunit is very similar in the two species. Because of the strong effect of resonance enhancement on CARS intensity, this statement is limited in the sense that it refers to that fraction of the chromoproteins whose contribution to the total absorbance at the pump wavelength is highest. For this and other reasons, we postulated that, in the CARS spectra of PC trimers, the signal contribution should originate predominantly from the $\beta 84$ chromophores (with smaller contributions from $\alpha 84$ ) if the pump wavelength is chosen to be $630 \mathrm{~nm}$ or higher [19]. Although X-ray analysis indicates that the geometries of the PCB chromophores of the $\beta$-subunits are fairly similar in PEC and PC from Mastigocladus laminosus, we nevertheless find pronounced differences in the CARS spectra of PEC trimers and PC trimers recorded with a pump wavelength of $630 \mathrm{~nm}$ [14]. We could claim that, due to the effect of resonance enhancement, for PEC the signal contributions originate exclusively from the $\beta$-chromophores (because of the $50 \mathrm{~nm}$ hypsochromic shift of the absorption of the $\alpha$-chromophore), whereas in PC there is a superposition of the signals of both $\alpha 84$ and $\beta 84$. On the other hand, it has been proposed that the order of the excited states of $\beta 84$ and $\beta 155$ chromophores is reversed in trimers of PEC compared with PC [13]. Such an exchange of level order would have the effect that signal contributions from $\beta 155$ would be enhanced in PEC relative to PC for energetic reasons (resonance enhancement). However, it is not clear whether the scattering cross-section of the $\beta 155$ chromophore is large, since even the 585 $\mathrm{nm}$ spectra of $\mathrm{PC}$ provide no evidence for contributions from this chromophore.

A recent analysis of the absorption and circular dichroism (CD) spectra of PEC from Mastigocladus laminosus [20], using chemical modification of the chromophores, indicates that the absorption maximum of the $\beta 155$ chromophore is located around $585 \mathrm{~nm}$ in both monomers and trimers, whereas the absorption maximum of the $\beta 84$ chromophore shifts hypsochromically from about $607 \mathrm{~nm}$ in the monomer to about $585 \mathrm{~nm}$ in the trimer (see Fig. 1). However, no decision was possible as to which of the two chromophores has the higher excited state in the trimer. Therefore an exchange in level order compared with PC cannot be excluded. In view of the similarity between the CARS spectra of PEC in the two organisms, whatever conclusion is drawn for PEC from Mastigocladus laminosus should also be valid for Westiellopsis prolifica.

The differences between the CARS spectra of PEC trimers for pump wavelengths of 585 and $630 \mathrm{~nm}$ have been discussed elsewhere for Mastigocladus laminosus [14]. By comparison with the spectra of trimers, which were subject to deuterium exchange, and the results of a normal coordinate calculation using the geometry of the PCB chromophores of PC provided by X-ray spectroscopy [5], it is possible to suggest assignments for several of the observed bands. The band pair around $1265 / 1275 \mathrm{~cm}^{-1}$ was assigned to $\mathrm{C} 5 \mathrm{H} / \mathrm{N} 21 \mathrm{H}$ bending in the $\beta$-chromophores in accordance with its disappearance in the $585 \mathrm{~nm}$ spectrum. In the $\alpha$-chromophore, this part of the molecule should give rise to the strong band around $1240 \mathrm{~cm}^{-1}$.

However, the model calculations show that all normal coordinates are strongly delocalized, although sometimes only over one or two of the tetrapyrrole rings and/or the adjacent methine bridges. The bands observed in the $\beta$-chromophores around 1250 and 1350 $\mathrm{cm}^{-1}$ are, for example, located in ring $\mathrm{D}(\mathrm{N} 23 \mathrm{H})$ and the adjacent methine bridge $(\mathrm{C} 15 \mathrm{H})$. The strong band around $1650 \mathrm{~cm}^{-1}$ is assigned to $\mathrm{C} 15=\mathrm{C} 16$ stretch coupled with $\mathrm{CC}$ and $\mathrm{CO}$ stretching vibrations in ring D. Since all these bands exhibit either strong changes 
in intensity or shifts in frequency with a change in pump wavelength from 630 to $585 \mathrm{~nm}$, we conclude that the $\alpha$ - and $\beta$-chromophores differ in geometrical and/or electronic structure around the C15 methine bridge in the two species. Of course we could speculate that all $\alpha$-chromophores change their geometry by $Z$ to $E$ isomerization because of the high dose of $585 \mathrm{~nm}$ radiation absorbed during the CARS experiment. However, this hypothesis seems unlikely in view of the small permanent changes seen in the UV-visible absorption spectra at the end of the CARS measurement and the fact that the photoinduced $Z$ to $E$ isomerization is reversed only very slowly in the dark. The small permanent changes caused by prolonged irradiation with pump and probe beams are most probably due to alternative reactions which lead to products whose absorption maxima are also around $570 \mathrm{~nm}$ (and therefore experience resonance enhancement). A second type of photochemistry leading mainly to band broadening has been described for PEC from Mastigocladus laminosus [21]. Furthermore, because the $510 \mathrm{~nm}$ absorbing isomeric species should not experience a large resonance enhancement, the $585 \mathrm{~nm}$ spectra must characterize the predominant isomeric form(s) which absorbs around $585 \mathrm{~nm}$ and should contain no contribution originating from the $510 \mathrm{~nm}$ absorbing species.

The differences observed in the double bond stretching region in the 585 and $630 \mathrm{~nm}$ spectra are usually taken as clear evidence that the $\alpha$-chromophore has a geometry around C15 which is different from that of the $\beta$-chromophores. Unfortunately, it cannot be decided on the basis of the rough model calculations whether the differences in chromophore-protein arrangement of $\alpha$ - and $\beta$-chromophores manifested in the $X$-ray structures $[4,5]$ are sufficient to explain the band shifts. Alternatively, it may be that, during the crystallization process, one arrangement is stabilized which is not the most stable in room temperature solution. Moreover, model calculations by Scharnagl and Fischer [22] indicate that the presence of solvent (water) is decisive for the most stable chromophore arrangement in $\alpha$-PEC. In an earlier publication, Scharnagl and Schneider [23] showed that the absorption maximum of the $\alpha 84$ chromophore in PC varied between 550 and $620 \mathrm{~nm}$ for different tautomeric forms of the propionic acid side-chains and arginine residues located near the central methine bridge. Similar effects were found for the two $\beta$-chromophores [24]. The coulombic interaction, with the distribution of uncompensated partial charges on the polar residues, polarizes the $\pi$ electron system of the bilin chromophore and leads to significant changes in $\pi$-bond order in both the ground and excited states. As a consequence of changes in $\pi$ bond order in the electronic ground state $S_{0}$, the vibrational frequencies can be changed without changing the geometry. Changes in the bond order of the first excited state $S_{1}$ lead to changes in the Franck-Condon factors for the $S_{0} \rightarrow S_{1}$ transition and, concomitantly, to variations in resonance Raman band intensities.

The weakness of the CARS spectrum of $\operatorname{PEC}(X)$ is due in part to the experimental conditions (see above). There could, however, be a second reason, namely the photoinduced conversion of many chromophores into chromophore-protein arrangements which are not in resonance with the $580 \mathrm{~nm}$ pump radiation. Alternatively, if the chromophores adopt too many different geometries, the concentration of any one arrangement will be too low to produce a spectrum with sufficiently high signal-to-noise or band-to-background ratio. The coincidence of frequencies in the $\mathrm{PEC}(\mathrm{X})$ spectrum and the $630 \mathrm{~nm}$ trimer spectrum is surprising. A possible explanation is that there is a predominant contribution from PVB chromophores, in which the (electronic) structure is similar to that of the $\beta 155$ chromophore in PEC. The contribution of the latter must prevail in the $630 \mathrm{~nm}$ spectrum, whereas the modified (compared with PC and PEC monomers) $\beta 84$ chromophores dominate in the $585 \mathrm{~nm}$ spectrum.

The large similarity in chromophore-protein arrangement, deduced from the agreement between the CARS spectra of PEC from the two organisms, does not necessarily mean that the distances between the $\beta$-chromophores of the various monomeric units in the trimer and their relative orientations are the same in Mastigocladus laminosus and Westiellopsis prolifica. Nevertheless, it is a good hypothesis to begin the interpretation of the time-resolved fluorescence data presented in Tables 1-3. In agreement with early results on PEC trimers of Mastigocladus laminosus [12], we interpret the third component with a derived decay time of between 1 and 1.3 ns as the lifetime of the terminal emitter, a PCB chromophore. This interpretation is in accordance with the increase in relative amplitude for detection at longer wavelengths. The very fast rising component (less than 20 ps) must be assigned to those PVB chromophores in the $\alpha$-subunit which are subject to fast energy transfer to the ensemble of $\beta$-chromophores. Thus the first decay component $\tau_{2}$ (200-300 ps) must be related to the energy transfer between the two types of $\beta$-chromophore. Since little is known about the exact excitation energy of these two chromophores, we cannot decide whether this lifetime represents the inverse of the energy transfer rate from the higher lying state or an equilibration process including back energy transfer (see also discussion above on the order of excited states). The last component (approximately $2.5 \mathrm{~ns}$ ) must be assigned to impurities or photo(oxidation) products. It is very unlikely that the structure of these long-lived species is close to that of the PCB chromophores. PCB chromophores exhibit a fluorescence decay time of about $1.5 \mathrm{~ns}$ if they are in the fully extended conformation, 
e.g. in the native state of PC $[2,11,12]$. The semiextended and cyclic helical forms, present in the denatured state or some butterfly biliproteins [25], show fluorescence decay times which are much shorter.

Hucke et al. [13] have recently studied energy transfer in trimers of PEC of Mastigocladus laminosus applying a two-colour, pump-probe technique with excitation at $575 \mathrm{~nm}$. For a probe wavelength of $578 \mathrm{~nm}$, they found a recovery of the initial bleaching with a time constant of $585 \pm 40 \mathrm{fs}$. For a stepwise increase in the detection wavelength up to $617 \mathrm{~nm}$, the bleaching showed a delayed onset with a rise time between 380 and 580 fs. All other isotropic kinetic components were longer than $100 \mathrm{ps}$. As in other phycobiliproteins [26,27], the ultrafast component is discussed in terms of energy transfer from $\alpha 84$ PVB to the $\beta$-chromophores of the neighbouring subunit. The second component, which showed a scatter in decay time between 110 and 330 ps, was related to energy transfer between the $\beta$ chromophores. In summary, we can say that the decay kinetics of the trimers of Mastigocladus laminosus and Westiellopsis prolifica are qualitatively similar, as expected on the basis of a similar chromophore-protein arrangement.

In the monomeric unit which contains the three nonequivalent chromophores, we would expect, at most, three exponentially decaying components. If the system exhibited no heterogeneity, the fits of the fluorescence decay curves recorded at different observation wavelengths should result in the same lifetimes, but the components should have different amplitudes. If we assume again, as mentioned above, that the fourth component is due to impurities or photo(oxidation) products, we have the correct number of components, but the decay times vary with detection wavelength.

The slowest remaining component with a decay time of approximately $1.3 \mathrm{~ns}$ must represent the lifetime of the terminal emitter. It may be slightly longer than in the trimers due to the lack of coupling between the three equivalent chromophores of neighbouring monomers. It would also be reasonable to assume that the second component (approximately $500 \mathrm{ps}$ for $\lambda \geqslant 610$ $\mathrm{nm}$ ) is longer than in the trimer (approximately 250 ps) because the route for energy transfer between $\beta 155$ and $\beta 84$ of neighbouring subunits is missing. Furthermore, this decay time should be longer than in PC trimers because the excited state of the PVB chromophore is energetically no longer in between the excited states of the two non-equivalent $\beta$-chromophores as is the case for the $\alpha$-PCB chromophore.

The first component with its large (relative) variation in decay time is puzzling. There are at least two different explanations. One possibility is that the energy transfer time between $\alpha$-and $\beta$-chromophores is $140 \mathrm{ps}$ in intact monomers (detection wavelengths, 610 and $620 \mathrm{~nm}$ ). Such an increase in transfer time compared with trimers is reasonable in view of the fact that, in trimers, the energy transfer between $\alpha$ - and $\beta$-chromophores is enhanced by the close proximity of $\alpha 84$ and $\beta 84$ chromophores in neighbouring monomers (see Fig. 1) [26]. Since, with detection at $590 \mathrm{~nm}$, the contribution of the terminal emitter to the fluorescence decay curve is only about $5 \%$, the two shorter lifetimes ( 0.24 and $0.8 \mathrm{~ns}$ ) could be related to the emission of $\alpha$-chromophores which do not participate in fast energy transfer, either because of large distances or because of a modified protein-chromophore arrangement. In any case, there should be a heterogeneity in chromophore geometry such that more than one decay time for this uncoupled chromophore can be rationalized.

On the other hand, a $30 \mathrm{ps}$ component with a relative amplitude of $50 \%$ is observed if the decay curve is recorded at $630 \mathrm{~nm}$. This wavelength corresponds to the emission maximum in the trimeric state in which energy transfer is very efficient. If, as the second possibility, this decay time is assigned to the energy transfer time between $\alpha$ - and $\beta$-chromophores in the intact monomer, we would expect a negative amplitude representing a rising component. The failure to detect such a rising component in the longer wavelength range indicates that very efficient energy transfer between the $\alpha$ - and $\beta$-chromophores is absent in the monomers. However, we cannot explain the 140 ps decay component for the measurements at 610 and $620 \mathrm{~nm}$ in this way. Since this wavelength regime corresponds to the overlap of PCB and PVB emission, it may well be that the $140 \mathrm{ps}$ component actually represents two components, one with a decay time of the order of $240 \mathrm{ps}$ and the other of the order of $30 \mathrm{ps}$. These components could then represent the decay time of structurally modified chromophores.

In denatured PC and chromopeptides, such short lifetimes have been recorded and assigned by comparison with the fluorescence kinetics of butterfly biliproteins with chromophores in the cyclic helical conformation [27] to PCB chromophores with such a geometry. The assumption that some of the PCB chromophores are in a partly relaxed conformation is also supported by the irradiation-induced changes observed in the UV-visible absorption spectra or, more precisely, by the decrease in the red absorption bands and increase in the near-UV-visible absorption. It has been stated previously that the photoreactions are more efficient for smaller aggregates. Because PEC from Westiellopsis prolifica is very photolabile in its monomeric state, we could not record CARS spectra with a pump wavelength of $585 \mathrm{~nm}$. Therefore some of the heterogeneity in the monomeric sample may be caused by the fairly weak excitation light used in the fluorescence measurements. The existence of a pronounced structural heterogeneity (which can be changed by excitation radiation) eliminates, in our opinion, the justification for the application 
of a global fitting procedure to improve the fitting parameters.

A well-established procedure to obtain an estimate of the intrinsic lifetime of a sensitizing chromophore is the isolation of this chromophore, e.g. in the form of a subunit where many of the energy transfer routes are no longer present. If $\mathrm{PEC}(\mathrm{X})$ actually represents the $\alpha$-subunit (the $N$-terminal sequence is equal to that of $\alpha$-PEC from other organisms [28]), its fluorescence kinetics may give important information on the lifetime of the $\alpha$-chromophore in various geometries and different chromophore-protein arrangements. However, as mentioned previously, $\operatorname{PEC}(\mathrm{X})$ is more photolabile than the monomer, and therefore it is not surprising that we need more than one exponential to fit the fluorescence decay curve.

With a detection wavelength of $590 \mathrm{~nm}$, the two major components exhibit lifetimes of 155 and 728 ps; both values are in fair agreement with the lifetimes derived under the same conditions for PEC monomers. This can be taken as evidence that these two components actually originate from two specific chromophoreprotein arrangements which are common to both PEC $(X)$ and PEC in the monomeric state. The fluorescence of the shorter lived state (fastest component) extends beyond $650 \mathrm{~nm}$, since at this wavelength the 180 ps component has a relative amplitude of more than $50 \%$. The emission of the second species is limited to the shorter wavelength region and is replaced at longer wavelength by the emission of a species with a lifetime of about $1.5 \mathrm{~ns}$. When the emission of this species is also present in the monomer fluorescence, it will be masked by the more intense emission of the PCB chromophores. This long-lived, long-wavelength emission may originate from a so-called $Z$ - $\alpha_{\text {II }}$ chromophore which has been shown to be a photoproduct in the $\alpha$-subunit of PEC from Mastigocladus laminosus. It is formed on oxidation of or reaction of mercurials with cysteines 98/99 and shows fluorescence around $640 \mathrm{~nm}$.

As yet, little is known about the conformation and fluorescence kinetics of the $\pi$-electron system of the PVB chromophore containing three conjugated pyrrole rings in the free state. Any further speculation about possible chromophore geometries or modifications of the chromophore structure is, therefore, not very useful at the present stage.

\section{Acknowledgements}

Financial support by the Deutsche Forschungsgemeinschaft (SFB 143, project A 1) and the Fonds der Chemie is gratefully acknowledged.

\section{Supplementary material available}

Tables containing all fitting parameters (frequencies $\nu_{\mathrm{r}}$, linewidths $\Gamma_{\mathrm{r}}$, relative intensities $A_{\mathrm{r}}=\left(R_{\mathrm{r}}^{2}+I_{\mathrm{r}}^{2}\right)^{1 / 2}$ and angles $\alpha_{\mathrm{r}}=\arctan I_{\mathrm{r}} / R_{\mathrm{r}}$ ) for all investigated samples and applied pump wavelengths are available on request to S. Schneider. The tables can be provided as printouts or on diskette.

\section{References}

[1] R. MacColl and D. Guard-Friar, Phycobiliproteins, CRC, Boca Raton, FL, 1987.

[2] H. Scheer, in C.A. Staehelin and C.J. Arntzen (eds.), Encyclopaedia of Plant Physiology, Vol. 19, Photosynthesis III, Springer, Berlin, 1986, pp. 327-337.

[3] P. Füglistaller, F. Suter and H. Zuber, The complete aminoacid sequence of both subunits of phycoerythrocyanin from the thermophilic cyanobacterium Mastigocladus laminosus, HoppeSeyler's Z. Physiol. Chem., 365 (1983) 1085-1096.

[4] T. Schirmer, W. Bode and R. Huber, Refined 3-dimensional structures of 2 cyanobacterial C-phycocyanins at 2.1 and 2.5 A resolution, J. Mol. BioL, 196 (1987) 677-695.

[5] N. Dürring, R. Huber, W. Bode, R. Ruembeli and H. Zuber, Refined 3-dimensional structure of phycoerythrocyanin from the cyanobacterium Mastigocladus laminosus at $2.7 \AA, J$. Mol. Biol., 211 (1990) 633-644.

[6] W. Rüdiger and F. Thuemmler, Phytochrom, Das Pigment der Pflanzen, Angew. Chem., 103 1242-1254; Angew. Chem. Int. Ed. Engl., 30 (1991) 1216-1228.

[7] S. Siebzehnrübl, R. Fischer, W. Kufer and H. Scheer, Photochemistry of phycobiliproteins: reciprocity of reversible photochemistry and aggregation in phycoerythrocyanin from Mastigocladus laminosus, Photochem. Photobiol., 49 (1989) 753-761.

[8] P.S. Maruthi Sai, S. Siebzehnrübl, S. Mahajan and H. Scheer, Phycoerythrocyanins from Westiellopsis prolifica and Notroc $r$ gulare. Characterization of the phycoviolobilin chromophore in both states, Photochem. Photobiol., 55 (1992) 119-124.

[9] P.S. Maruthi Sai, S. Siebzehnrübl, S. Mahajan and H. Scheer, Fluorescence and circular dichroism studies on the phycoerythrocyanins from the cyanobacterium Westiellopsis prolifica, Photochem. Photobiol, 57 (1993) 71-75.

[10] W. Kufer and G.S. Bjöm, Photochromism of the cyanobacteria light-harvesting biliprotein, phycoerythrocyanin, Physiol. Plant., 75 (1989) 389-394.

[11] P. Hefferle, M. Nies, W. Wehrmeyer and S. Schneider, Picosecond time-resolved fluorescence study of the antenna system isolated from Mastigocladus laminosus Cohn. II. Constituent biliproteins in various forms of aggregation, Photobiochem. Photobiophys., 5 (1983) 325-334.

[12] S. Schneider, P. Geiselhardt, T. Mindl and F. Dörr, Picosecond time-resolved polarized fluorescence decay of phycobilisomes and constituent biliproteins isolated from Mastigocladus laminosus, in M.E. Michel-Beyerle (ed.), Antennas and Reaction Centers of Photosymthetic Bacteria, Springer, Berlin, 1985, pp. 26-35.

[13] M. Hucke, G. Schweitzer, A.R. Holzwarth, W. Siedler and H. Zuber, Studies on chromophore coupling in isolated phycobiliproteins. IV. Femtosecond transfer absorption study of ultrafast excited state dynamics in trimeric phycoerythrocyanin complexes, Photochem. Photobiol., 57 (1993) 76-80.

[14] S. Schneider, C.-J. Prenzel, G. Brehm, L. Gottschalk, K.-H. Zhao and $H$. Scheer, Resonance-enhanced CARS spectroscopy of biliproteins. I. Reversible photochemistry in the $\alpha$-subunit 
of phycoerythrocyanin (Mastigocladus laminosus), Photochem. Photobiol, submitted for publication.

[15] R. MacColl, G. O'Connor, G. Croften and K. Csatorday, Phycoerythrocyanin; its spectroscopic behavior and properties, Photochem. Photobiol., 34 (1981) 719-723.

[16] M. Gahr, Untersuchung der Bildung und des Zerfalls von interund intramolekularen Styryl-Amin-Exciplexen, Ph.D. Thesis, Friedrich-Alexander-Universităt, Erlangen, 1993.

[17] C.-J. Prenzel, CARS-Studien der durch Aggregation induzierten Konformationsänderungen von Tetrapyrrolchromophoren in Biliproteinen, Ph.D. Thesis, Friedrich-Alexander-Universităt, Erlangen, 1993.

[18] H. Scheer, unpublished results.

[19] S. Schneider, C.-J. Prenzel, G. Brehm, P. Gedeck, P.S. Maruthi Sai, L. Gottschalk and H. Scheer, A comparison of phycocyanins from three different species of cyanobacteria employing resonance-enhanced coherent anti-Stokes Raman spectroscopy, Photochem. Photobiol., 57 (1993) 56-62.

[20] H. Scheer, unpublished results.

[21] Q. Hong, K.-H. Zhao and H. Scheer, Two different types of photochemistry in phycoerythrocyanin $\alpha$-subunit, Photochem. Photobiol, 58 (1993) 745-747.

[22] C. Scharnagl and S.F. Fischer, Reversible photochemistry in the $\alpha$-subunit of phycoerythrocyanin: characterization of chromophore and protein by molecular dynamics and quantum chemical calculations, Photochem. Photobiol., 57 (1993) 63-70.
[23] C. Scharnagl and S. Schneider, UV-visible absorption and circular dichroism spectra of the subunits of C-phycocyanin. I: Quantitative assessment of the effect of chromophore-protein interaction in the $\alpha$-subunit, J. Photochem. Photobiol. B: Biol., 3 (1989) 603-614.

[24] C. Scharnagl and S. Schneider, UV-visible absorption and circular dichroism spectra of the subunits of C-phycocyanin. II: A quantitative discussion of the chromophore-protein and chromophore-chromophore interactions in the $\beta$-subunit, $J$. Photochem. Photobiol. B: Biol., 8 (1991) 129-157.

[25] S. Schneider, F. Baumann, P. Geiselhardt, H. Kayser and H. Scheer, Biliproteins from butterfly Pieris brassicae studied by fluorescence and coherent anti-Stokes Raman spectroscopy, Photochem. Photobiol., 48 (1988) 239-242.

[26] K. Sauer and H. Scheer, Excitation transfer in C-phycocyanin. Forster transfer rate and exciton calculations based on new crystal structure data for $\mathrm{C}$-phycocyanin from $A$ Agmanellum quadruplicatum and Mastigocladus laminosus, Biochim. Biophys. Acta, 936 (1988) 157-170.

[27] A.V. Sharkov, I.V. Kryakov, E.V. Khoroshilov, P.G. Kryukov, R. Fischer, H. Scheer and T. Gillbro, Femtosecond energy transfer between chromophores in allophycocyanin trimers, Chem. Phys. Lett, 191 (1992) 633-638.

[28] F. Lottspeich, P.S. Maruthi Sai and H. Scheer, unpublished results. 